\title{
High-Resolution Health Monitoring of Track and Rail Systems with Fiber Optic Sensors and High-Frequency Multiplexed Readouts
}

\author{
Kyle Arakaki ${ }^{1}$, Ajay Raghavan $^{1}$, Andreas Schuh ${ }^{1}$ \\ ${ }^{1}$ Palo Alto Research Center, Palo Alto, CA, 94304, USA \\ Kyle.Arakaki@parc.com \\ Ajay.Raghavan@parc.com \\ Andreas.Schuh@parc.com
}

\begin{abstract}
Health monitoring of railway systems is critical for detecting incipient faults or degradation. In order to reliably do so, an effective monitoring system must be deployed to provide railroad operators with the highest level of operational awareness and safety. In this study, we explore the use of Fiber Bragg Gratings (FBGs) and a highresolution, low-cost optical readout developed at PARC to interrogate the acoustic emissions generated by a train-rail system. The proposed sensing configuration can allow for a scalable, low-cost, field-deployable solution that could enable near real-time monitoring of tracks and wheels. A proof-of-concept was demonstrated with a G-scale train-rail system with FBGs embedded within the ballast layer. Using PARC's wavelength shift detector, the acoustic emission signal was resolved in both the time and frequency domain. The findings of this work show promise that this could be a viable solution to deploy an optically-based health monitoring system for railroads.
\end{abstract}

\section{INTRODUCTION}

Structural failure of rails and wheels are some of the leading causes of derailments (Liu, Saat, and Barkan, 2012). These failures can develop due to insufficient maintenance, manufacture defects, the environment, and operating conditions (load, speed, etc.). In the U.S. alone, approximately $1 / 3^{\text {rd }}$ of railroad accidents each year occur due to broken rails (Mouwad, 2015). During the past 50 years, heavier axle loads and increased train traffic/speeds in the North American railroad industry have caused the wheel/rail contact environment to become even more strenuous. This has led to increased wear and rolling contact fatigue (RCF), decreased rail life, and higher maintenance costs. Magel (2011) reported that RCF costs the US railroad

Kyle Arakaki et al. This is an open-access article distributed under the terms of the Creative Commons Attribution 3.0 United States License, which permits unrestricted use, distribution, and reproduction in any medium, provided the original author and source are credited. industry over $\$ 300 \mathrm{M}$ annually. Worn rails can also considerably increase fuel consumption, braking distance, lateral forces, noise, and wheel wear rates. Reliable early detection of RCF can enable proactive actions and could prevent $>90 \%$ of the $100+$ rail accidents caused by RCF annually (Magel, 2011). Traditional methods of high mileage railway maintenance typically require the deployment of an inspection car, HiRail trucks, or maintenance technicians/operators manually inspecting tracks or wheels in the field. Developing a fiber-optic (FO) based distributive sensing system could allow for enhanced fault detection as well as real-time automated condition based maintenance. In this paper, we explore the use of a high-resolution, scalable, optical wayside detector to monitor early stage faults through acoustic emissions.

Previous published works have demonstrated the feasibility of using various fiber optic (FO) sensor technologies to detect failure modes in railways systems. Chuang, Hsu, and Young (2003) used FO sensors mounted directly on rails to demonstrate their utility for rail break and buckling detection in both laboratory and field settings. They demonstrated that FO sensors using optical time-domain reflectometry (OTDR) can be used for train presence, speed, weigh-in, and wheel flat detection. Adoption of this sensing method was challenged by the fibers becoming damaged due to events such as ballast dumping and plowing. Papp, Wiesmeyr, Litzenberger, Gam, \& Kropatsch (2016) reported that FO sensing using OTDR has an effective monitoring range of several tens of kilometers. Holcomb and Mauger (2013) explored the feasibility of using an underground FO cable below the track. The FO cable monitored the acoustic energy generated by the track as a train passed by. After analyzing the changes in acoustic signatures during a passing event, they were able to detect rail breaks up to 5 train passes in advance. However, they were unable to distinguish between rail breaks and joints. 
While FO sensors have been shown to be capable of detecting the formation of various failure modes, a complete end-to-end standoff FO rail monitoring system that can differentiate between rail features and incipient damage has yet to be implemented in the field. In addition, any distributed railway FO monitoring system will also require suitable low-cost readouts. We attempt to address some of the challenges previously mentioned to provide a viable solution for a FO-based, railway health monitoring system.

\section{BACKGROUND}

The optical sensing system is comprised of three vital components: a light source (typically infrared), an optical sensing structure, and an optical readout. In this section, we will focus on the sensing structure and optical readout.

\subsection{FBG sensors}

Fiber Bragg Gratings are structures in an optical core that are designed to reflect a very narrow band of light when the Bragg condition is satisfied (Hill \& Meltz, 1997). This structure is formed by scribing a periodic modulation in the core's refractive index. Any elongation or compression of this structure will result in a change in the Bragg condition, resulting in a shift in the wavelength reflected. The response of an FBG sensor to external stimuli, such as strain or temperature, can be determined by measuring the difference between the nominal wavelength prior to acting forces and the wavelength reflected at the time of stimuli.

One advantage of FBG sensors is that a single FO cable can have potentially hundreds of these structures embedded at different locations each designed to reflect a different wavelength or physically spaced out sufficiently to be able distinguish/time-gate their individual responses. Using such multiplexing techniques can enable distributive sensing of a large structure, such as a section of railway. In addition, the signals reflected by an FBG are much stronger than those produced in OTDR systems, allowing for greater sensitivity and distributed sensing across a FO cable $\sim 100 \mathrm{~km}$ in length (Fernandez-Vallejo \& Lopez-Amo, 2012).

While there are many characteristics of FBGs that make it ideal for distributive sensing, decoupling static strain responses or low-frequency vibrations from environmental effects using just a single FBG can be challenging, especially in fielded deployments. Numerous schemes have been explored to compensate for either temperature, humidity, or other environmental stimuli (Van Steenkiste \& Springer 1997). In this paper, we discuss the use of multiplexed FBGs as a means to monitor high frequency acoustic emissions generated in a train-railway system to reliably detect and distinguish failure modes.

\subsection{FO Readout}

Field deployment of wavelength-encoded sensors have been challenged by the cost, size, weight, and resolution of the interrogation unit. In a step towards addressing this issue, PARC has developed a low-cost, compact, and sensitive wavelength shift detector (Schmidt, Kiesel, Mohta, and Johnson, 2012). The optical sensing system consists of a linear variable filter (LVF) and an array of photodiode detectors. The LVF essentially converts the incoming light into a spatial intensity distribution across a pair of photodiodes. As reported in Schuh, Hegyi, Raghavan, Lochbaum, Schwartz, and Kiesel (2015), this readout could obtain a sensitivity of less than $90 \mathrm{fm}$ at frequencies up to $80 \mathrm{kHz}(>10 \mathrm{x}$ the state-of-the-art (SOTA)), as well as be scaled up to monitor many multiplexed sensors (U.S. Patent No. 20160018319 A1, 2016).

\section{EXPERIMENTAL}

Experiments were carried out in a laboratory setting using a 1:22 G-scale train system, shown in Figure 1a. A circular loop of steel track was assembled on a layer of ballast (also $\mathrm{G}$-scale). The load on the tracks consisted of an electric

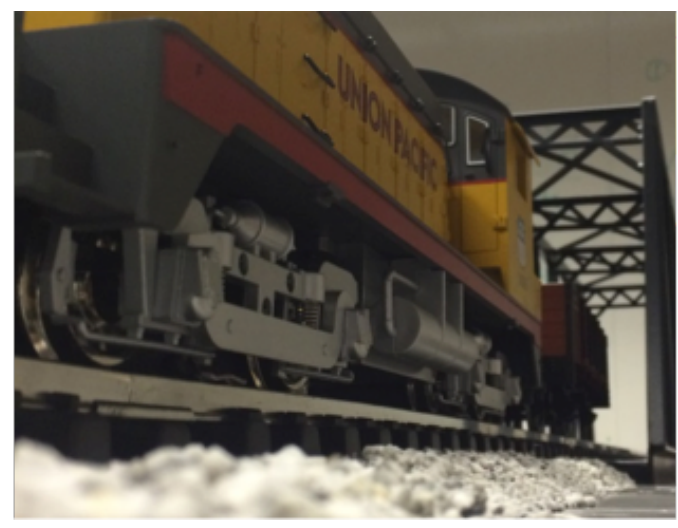

a)

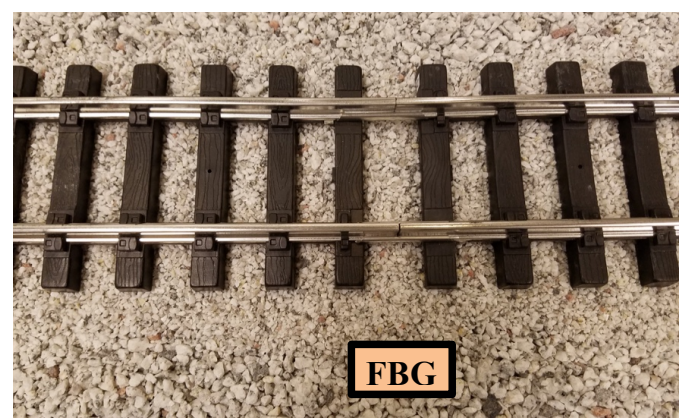

b)

Figure 1. a) Photo of G-scale rail system used for initial experiments; b) FBG sensor placed 2" away from track 
locomotive and a carrier wagon in which weights were placed into. An FBG sensor was placed in the ballast layer 2 " away from the tracks as shown in Figure 1b. In a fullscale train system, this would correspond to a sensor placed $\sim 3.7$ ' away from the tracks. This distance is comparable to the distance that Holcomb and Mauger (2013) used in their experiments. The FBG was then connected to PARC's single channel, high-frequency optical readout. All signals here were recorded at a sampling rate of $1 \mathrm{kHz}$. Lastly, contributions from environmental temperature and humidity fluctuations were assumed to be negligible in the case of high frequency acoustic emissions. This is a reasonable assumption as these fluctuations tend to occur at much lower frequencies $(<1 \mathrm{~Hz})$.

\subsection{Test cases}

\subsubsection{Rail fractures}

To simulate a rail fracture in this scaled down system, a gap of $\sim 2-3 \mathrm{~mm}$ was introduced between two sections of track. The purpose of these measurements was to show that our sensing configuration could differentiate between a rail joint (healthy condition) and a rail fracture. This was one of the challenges cited by Holcomb and Mauger (2013).

\subsubsection{Surface abrasion}

Surface abrasion was introduced by rough filing throughout one section of track $(\sim 1 \mathrm{ft}$.). The FBG sensor was placed adjacent to this section of track.

\subsubsection{Wheel flats}

There are several conditions that lead to a wheel being considered condemnable. One condition is the formation of a slid flat of over 2 inches in length (FRA Railroad Locomotive Safety Standards, 2016). To simulate this fault case, one of the wheels was machined with a flat spot measuring $\sim 3.5 \mathrm{~mm}$ in length (Figure 2).

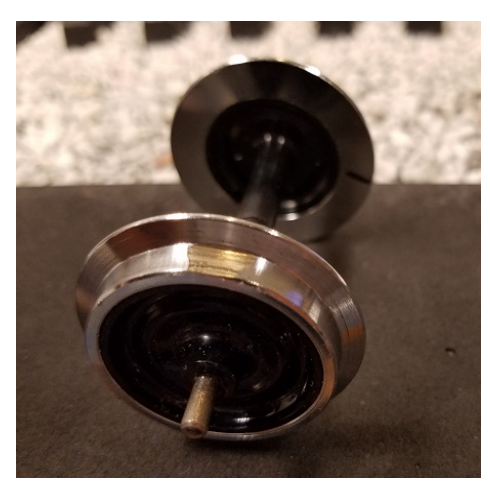

Figure 2. Axle with wheel flat

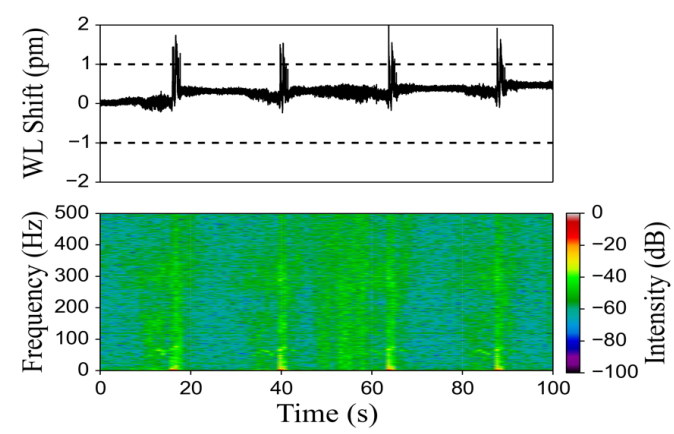

a)

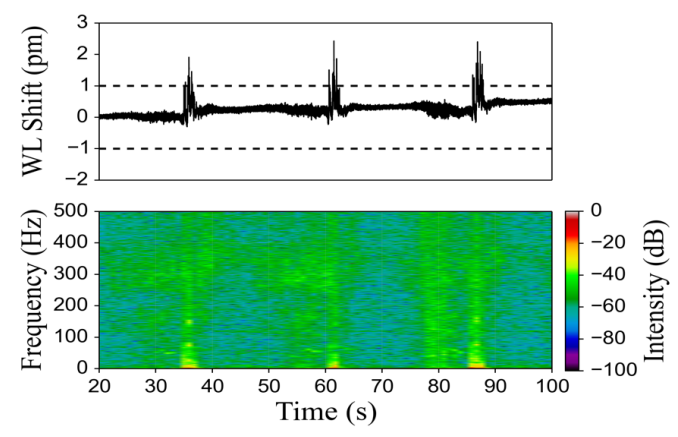

b)

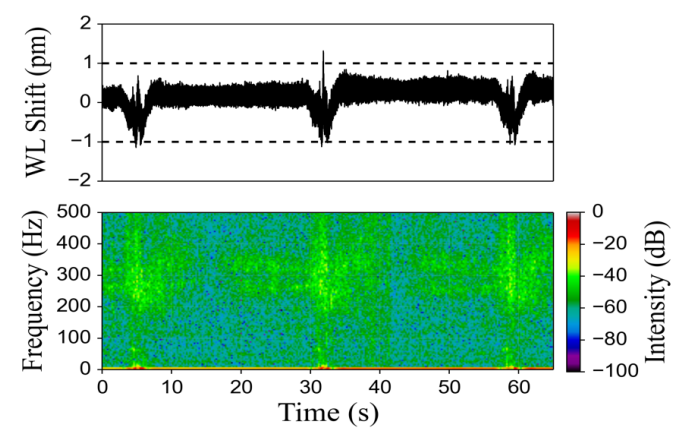

c)

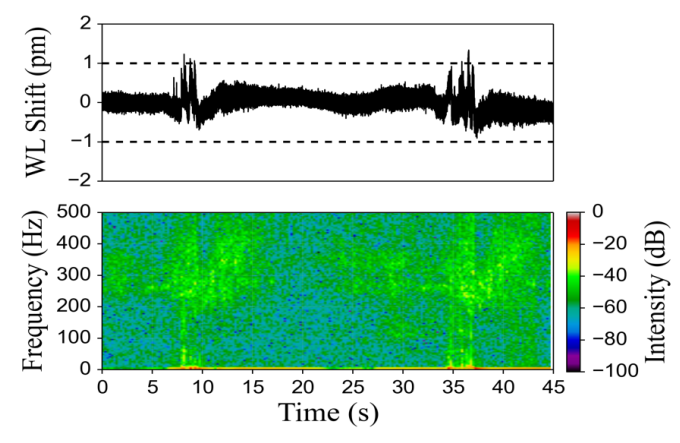

d)

Figure 3. Acoustic emissions from FBG and corresponding spectrogram as train passes for a) healthy track under no load; b) health track with load; c) healthy track with load removed (zoomed); d) simulated rail fracture. The dotted lines refer to current state-of the-art (SOTA) sensitivities. 


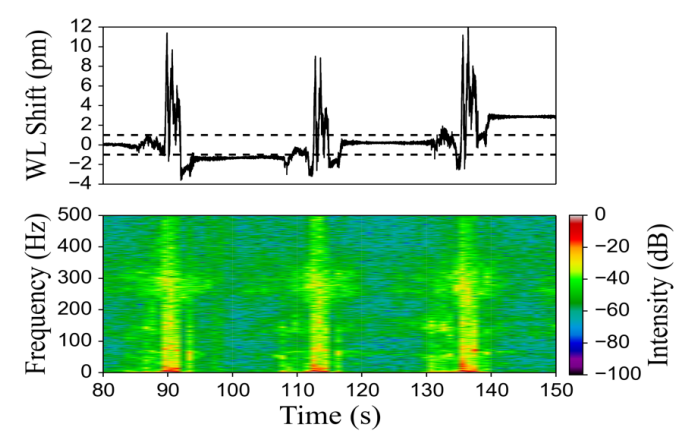

a)

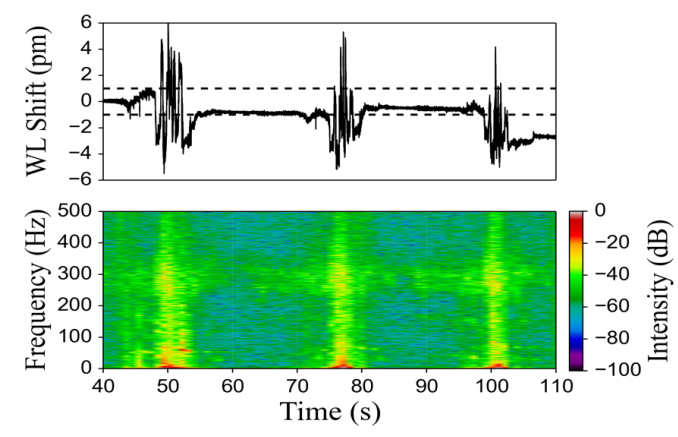

b)

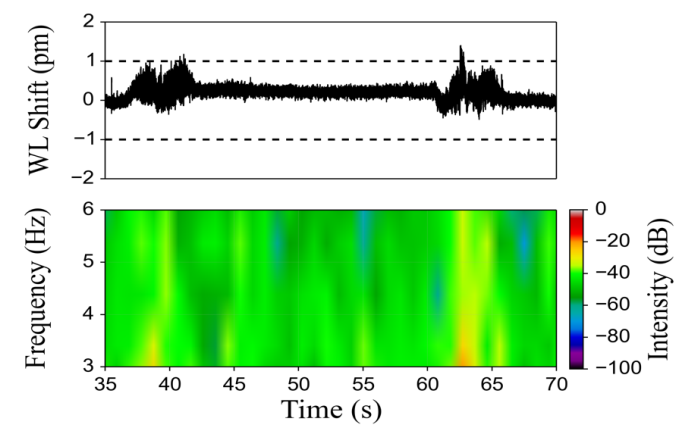

c)

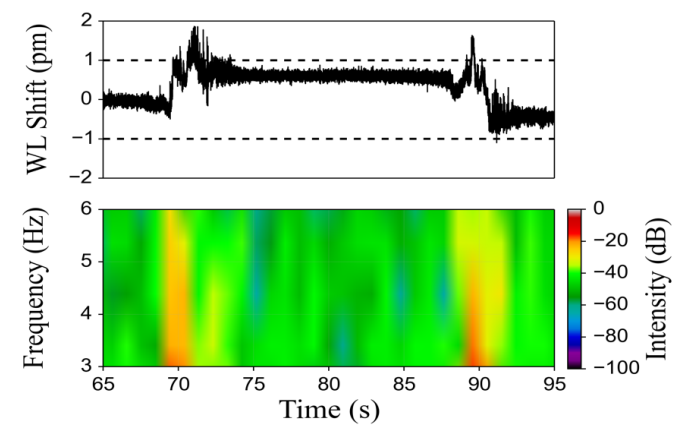

d)

Figure 4. Acoustic emissions from FBG sensor and corresponding spectrograms as train passes with a) no load and b) load, both for track with wear damage. Signals and spectrograms from c) a healthy system and d) train with wheel flat are zoomed in around $5 \mathrm{~Hz}$ frequency band.

\section{Results}

\subsection{Healthy rail under various load conditions}

As shown in Figure $3 a$ and $3 b$, the magnitude of wavelength shift as the train passes in close proximity to the FBG sensor does not vary much with load. If the FBG were attached directly to the rails, a large wavelength shift on the order of tens of picometers would have been measured corresponding to the weight of the train. However, since the FBG was placed in the ballast away from the rail, this strain has been dispersed throughout the ballast material. The frequency content of these measurements is concentrated between $5-50 \mathrm{~Hz}$ and $200-500 \mathrm{~Hz}$. Similar frequency bands were measured from a three-piece bogie by Shahidi, Maraini, and Hopkins (2016), for their railcar diagnostics.

\subsection{Damaged rail}

For both the rail fracture and corrugation simulations, a change in both the time and frequency domain signals were observed. When comparing a healthy track (Figure 3c) and a track with a simulated fracture (Figure 3d), we observed a response from a wider range of frequency bands, particularly between $50-150 \mathrm{~Hz}$. Most notable are the increased number of time-domain peaks - corresponding to the number of total wheels passing over the fracture. The acoustic emission response is considerably stronger for the case of track surface damage (Figure $4 \mathrm{a}$ and $4 \mathrm{~b}$ ).

\subsection{Wheel flats}

Measurements from this fault case suggest that wheel flats can be extracted from acoustic emissions. Similar to the case of the rail fracture, distinct peaks could be discerned in the time-domain signal (Figure 4d) compared to measurements from the healthy case (Figure 4c). Of particular interest for this case is the $5 \mathrm{~Hz}$ frequency band. This band corresponds well with the periodicity of contact between the wheel flat and rail with the train's velocity.

\section{Conclusion}

The railroad industry is facing a growing need for more advanced methods of health monitoring to detect and characterize system faults and degradation. While current on-site inspection techniques are somewhat capable of detecting developing fault modes in rails, the detection rate at a single location in a track is only as good as the frequency at which the inspection occurs at that site. Based on the findings of this paper, and previous works, fiber optic sensors can provide high-resolution data for incipient fault detection. While a single FO cable can provide pointsensing capabilities across many kilometers of railroad track, challenges such as, cost and robustness, have proven difficult to overcome. In a step towards addressing the issue of robustness, we explored moving the fiber optic sensors away from the rail, thus, avoiding damaging events such as 
ballast dumping. By using PARC's multiplexed wavelength shift detector, we were able to resolve the sub-picometer acoustic emission signals from a scaled down train-railballast system. Innovative time-multiplexing schemes that are focused selectively on FBG sensors immediately adjacent to the passing train can enable low-cost scale-up of this system for wayside monitoring of large lengths of rails. Thus, this method of high-resolution monitoring shows promise for enabling true condition-based maintenance in railroad systems. Validation of these results and integration with diagnosis, prognosis, and planning algorithms that can accurately locate, classify, quantify, predict, and plan operations around these monitored track conditions in fullscale systems would be next steps towards fielded deployment.

\section{ACKNOWLEDGEMENTS}

The authors acknowledge discussions with Anurag Ganguli, Parham Shahidi, and Peter Kiesel at PARC for this work.

\section{REFERENCES}

Chuang S. L., Hsu A., \& Young, E. (2003). Fiber Optical Sensors For High-Speed Rail Applications (Report No. HSR IDEA Project 19). Washington, DC: Transportation Research Board.

Fernandez-Vallejo, M., \& Lopez-Amo, M. (2012). Optical Fiber Networks for Remote Fiber Optic Sensors. Sensors (Basel, Switzerland), 12(4), 39293951. http://doi.org/10.3390/s120403929

FRA Railroad Locomotive Safety Standards, 49 C.F.R. § 229.75 (2016).

Heygi, A., Kiesel, P., \& Raghavan, A. (2016). U.S. Patent No. 20160018319 A1. Washington, DC: U.S. Patent and Trademark Office.

Hill, K.O., Meltz, G., (1997), Fiber Bragg Grating Technology Fundamentals and Overview, Journal of Lightwave Technology. 15 (8), pp 1263-1276

Holcomb, M. D., Mauger, W. D. (2013). Feasibility Study of Fiber-Optic Technology for Broken Rail Detection
(Report No. DOT/FRA/ORD-13/44). Washington, DC: U.S. Federal Railroad Administration

Liu X., Saat, M., \& Barkan, C. (2012). Analysis of causes of major train derailment and their effect on accident rates, Transportation Research Record: Journal of the Transportation Research Board, 2289: 154-163, doi:10.3141/2289-20

Magel, E. E., (2011). U.S. Department of Transportation, Federal Rolling Contact Fatigue: A Comprehensive Review. (Report No. DOT/FRA/ORD-11/24). Washington, DC: U.S. Federal Railroad Administration

Mouwad J. (2015, October 9). Railroad agency details new rail-track standards. New York Times, retrieved from http://www.nytimes.com

Papp, A., Wiesmeyr, C., Litzenberger, M., Garn, H., \& Kropatsch, W. (2016). A real-time algorithm for train position monitoring using optical time-domain reflectometry, 2016 IEEE International Conference on Intelligent Rail Transportation (ICIRT), pp. 89-93. doi:10.1109/ICIRT.2016.7588715

Schmidt, O., Kiesel, P., Mohta, S., \& Johnson, N. M. (2007). Resolving pm wavelength shifts in optical sensing. Applied Physics B. 86 (4), pp 593-600. doi:10.1007/s00340-006-2456-y

Shahidi, P., Maraini, D., \& Hopkins, B. (2016). Railcar Diagnostics Using Minimal-Redundancy MaximumRelevance Feature Selection and Support Vector Machine Classificiation. International Journal of Prognostics and Health Management. 7 (Special Issue Big Data Analytics). Retrieved from https://www.phmsociety.org/

Schuh, A., Hegyi, A., Raghavan, A., Lochbaum, A., Schwartz, J., \& Kiesel, P. (2015). High-resolution, high-frequency wavelength shift detection of optical signals with low-cost, compact readouts. Proc. SPIE, Fiber Optic Sensors and Applications XII. 9480, doi: $10.1117 / 12.217$

Van Steenkiste, R. J., Springer, G. S. (1996). Strain and Temperature Measurement with Fiber Optic Sensors. Lancaster, Pennsylvania: Technomic Publishing Company, Inc. 